\title{
THE MURBURN PRECEPTS FOR PHOTORECEPTION
}

\author{
Kelath Murali Manoj* and Vivian David Jacob \\ Satyamjayatu: The Science \& Ethics Foundation, Shoranur, Palakkad District, Kerala, India
}

\begin{abstract}
Murburn concept is a redox mechanistic scheme involving interactive equilibriums of discretized or organized protein/substrate molecules, unbound ions and radicals (or reactive species); which may afford selective/specific electron transfers, particularly at phospholipid interfaces. Earlier, we have applied murburn concept to provide thermodynamically and kinetically viable explanations for various physiological/bioenergetic routines like xenobiotic metabolism, unusual dose responses, aerobic respiration, thermogenesis, homeostasis, trans-membrane potential, oxygenic photosynthesis, etc. While proposing the murburn model for photophosphorylation, we had projected that the murburn mechanism could also be relevant for photoreception physiology. Herein, we expand on this aspect and present the basic scheme and evidence in support of the murburn model of photoreception, with retinal/opsin as the salient photon-impingement response-transducing element. In alignment with our earlier murburn schemes, we propose that diffusible reactive oxygen species (DROS, as exemplified by superoxide, which is currently deemed a toxic product of all-trans retinal and NADPH oxidase interactions) is produced in rod/cone cells upon photoactivation and it plays a crucial role in the visual cycle. This is supported by several facts: (i) Layers of photoreceptive neural cells precede the rod/cone cells (with respect to the presentation to oncoming light ray/photon), (ii) There exists high oxygen demand in the retina, (iii) Copious DROS are detected in functional retina, (iv) $N A D(P) H /$ reductase is involved in the cycle, and (v) Events occur at sub-micrometer dimensioned phospholipid disks stacked to stabilize DROS and minimize free protons (quite like the thylakoids that harbor carotenoids in chloroplasts and cyanobacteria). In the new scheme, photo-electric activation leads to charge separation and hyperpolarization in rod/cone cells due to negative charge build-up. This electro-chemical signaling serves as the front-runner to trigger an action potential relay along a neuron and the superoxide mediated phosphorylation of GDP bound to transducin serves as the initiator of signal transduction cascade. The newly proposed scheme allows a simple electrical connectivity of the retina-photoreceptors with the brain via the optic nerve, is anatomically correlated with the structure and resolution of the retina, and is kinetically viable. Biomed Rev 2020; 31: 67-74
\end{abstract}

Keywords: photoreception; signal transduction; rhodopsin; retina; vision; murburn concept; retinal

\footnotetext{
*Correspondence to: Dr Kelath Murali Manoj, Satyamjayatu: The Science \& Ethics Foundation,
} Shoranur-2 (PO), Palakkad District, Kerala, India Email: satyamjayatu@yahoo.com; (ORCID: 0000-0003-4515-994X) 


\section{INTRODUCTION}

Photoreception is a necessity for several living beings, from microbes to man. The physiology of vision has been investigated extensively and significant advances have been made towards understanding the structural features of retina and molecular aspects of vision $(1,2)$. Herein, with a skeptic's viewpoint, we revisit the prevailing perceptions regarding the phenomenological and mechanistic aspects of the physicochemical processes that transpire at/around the retina. In a related context, we had recently dissected the standing explanations for the light reaction of photosynthesis, and provided alternative explanations based on murburn concept. While doing so, we had projected that some aspects of the light-carotenoid-DROS (diffusible reactive oxygen species) interactive paradigm we had proposed within chloroplasts could also be relevant to the photoreception mechanistic cycle (3). This manuscript presents a succinct mechanistic outlay of the physico-chemical transduction processes involved in photoreception at retina based on murburn concept. We focus on the physiological processes involved in resolution and depth perception in monochromatic vision, with retinal/opsin as the pigment/protein of interest.

\section{A BRIEF SNAPSHOT OF THE CURRENT PERCEPTIONS ON THE VISUAL CYCLE}

The highlights of histological structure and dimensional features of the various domains of human retina are presented in Figure 1 (4). In Figure 2, we present a schematic of a rod cell and a molecular snapshot of the visual cycle and signal transductions that transpire at the Rod Outer Segments (ROS) and Retinal Pigmented Epithelium (RPE) (5-7). The hot-seat of visual cycle is at the apical disks of ROS, wherein the light-induced conversion of opsin-bound 11-cis retinal to all-trans retinal (Figure 2 ) occurs. This leads to the retinoid's dissociation from the rhodopsin protein. This step is supposed to trigger membranebound opsin GPCR (transducin), resulting in phosphorylationphosphodiesterase cascades and ion channel manipulations, etc. These trigger an action potential along neuronal axons of the retina, which is in turn relayed to the optic nerve. Meanwhile, the detached all-trans retinal is recycled via the respective retinols (and their esters) at the RPE and returned back to the ROS-disks for once again going through the visual cycle.

\section{BASIC ASSUMPTIONS FOR ENABLING DYNAMIC PHYSIOLOGICAL VISION}

Since it is surely known that an inverted and real image is

\section{SAMPLES OF SOME CELL TYPES OF RETINA}
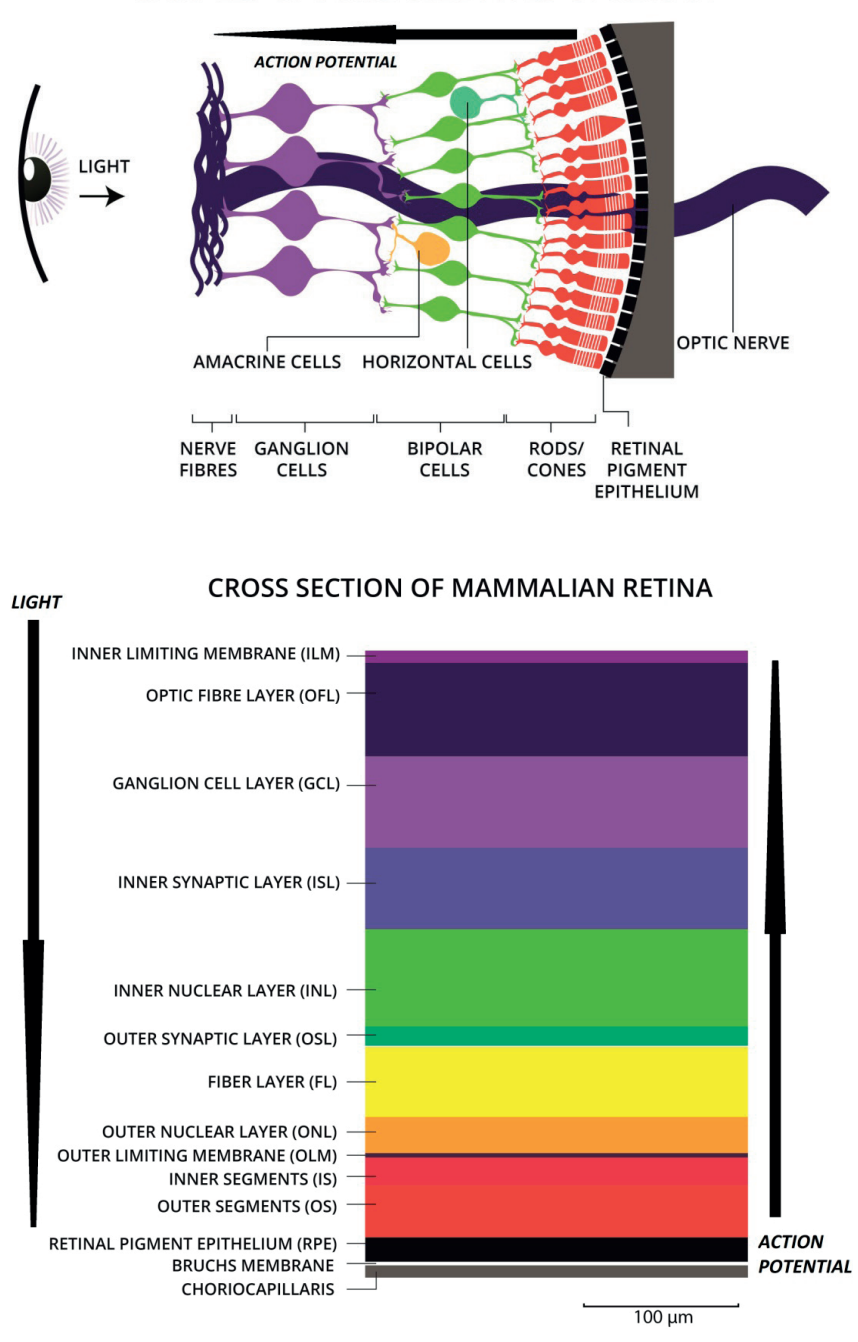

Figure 1: Structural and dimensional features of a human retina, as gauged from a cross-section. (Unlike the top panel that is not drawn to scale, the bottom panel shows that retina is 400 microns thick. The directions of light impingement and propagation of action potential are also shown. Coloring is merely to demarcate the layers.)

formed on the concave surface of retina, the retinal machinery must possess the spatial acuity to distinguish two distinct dots (or points). The optic nerves must receive/take signals that can differentiate between two nearby signals within $1 / 60^{\text {th }}$ of a second (which is the fastest frame rate possible with human eyes, as estimated by experts). That is, for continuous/dynamic vision, besides high spatial resolution, high temporal signal collection rate and photoreceptor regeneration rate must be availed for enabling a seamless processing of the physico- 

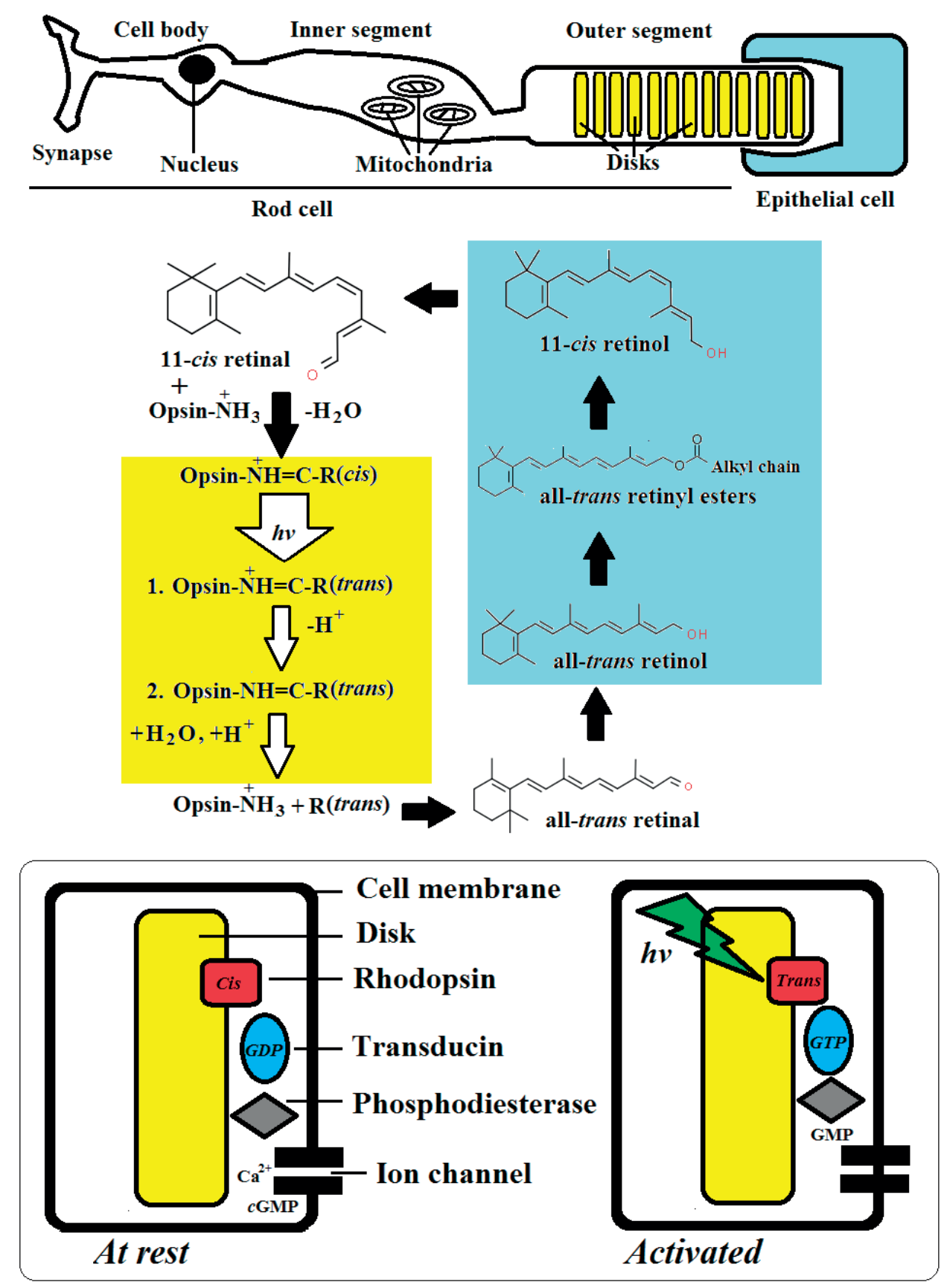

Figure 2: The structure of a rod cell and pigmented epithelium cell (the center-stage of visual cycle, top), the molecular/reaction details of the visual cycle (middle) and the cascades/changes initiated by a photon striking rhodopsin (bottom). Binding of 11-cis retinal to opsin creates the photoactive rhodopsin and this process involves the formation of a Schiff's base (and release of a water molecule) tethering of the pigment with the protein. The reactions highlighted in yellow occur within rod cell disks and form the crux of the transduction of an impinging photon, via steps that afford the relay of an electro-chemical signal to the optic nerve. Reactions highlighted in turquoise occur within RPE's endoplasmic reticulum. Structures 1 and 2 are bathorhodopsin and metarhodopsin, respectively and $R$ stands for retinal. Activation by light triggers transducin to bind to GTP and this protein activates phosphodiesterase, which cleaves cyclic GMP (cGMP). Since cGMP is needed to keep the calcium-specific channel open, this outcome resulting from light impingement of rhodopsin initiates an action potential that is relayed to neurons. For simplicity, several other ions and channels and proteins involved are not shown. 
chemical transduction process. Most importantly, there should be at least two electro-chemical events per receptor cycle: (i) an event that could enable the opsin (or the relevant retinal element) to convert the physical energy or impact of a photon into an electrical signal (that could be picked up or relayed to the neurons/optic nerve), and (ii) another event that could enable the system to reinstate itself to the excitable step from the post-discharged state. That is, post the first step, a neuron could be triggered and an action potential ripple could conduct along its axon and then, the regeneration process could take place.

\section{SOME CRITICAL QUERIES}

Literature documents several unsolved aspects regarding the physiology of vision (8). Here are some that we would like to highlight:

1. If the primary photoreceptive-excitation event occurs after a photon traverses almost $\sim 400 \mu \mathrm{m}\left(\sim 10^{3}\right.$ times the wavelength of an incident light ray of say, $400 \mathrm{~nm}$ ) into the retina's thickness (Figure 1), how can such an incident photon convey spatial information that could be constructed into a factual image? The photon that would reach an opsin at a given rod cell would not be merely transmitted light. Multitudes of intervening stacked/layered structures and various cells (Figure 1) would introduce secondary phenomena along the light's path within the retina, like: refraction, interference, diffraction, scattering, absorption-emission, reflection, etc., which would render the photon impingement at the ROS region "informatively less useful" for the construction of a realistic mage. If the present perception (that visual cycle is initiated at the ROS region) were true, then one would expect evolution to reverse the order of cells and the orientation of the relevant rod cells and neural fibers, as shown in Figure 3.

2. Birds have a retina in which the proximal retinal surface is not innervated/inundated with blood vessels, but humans' eyes are innervated/inundated at both proximal (concave side) and distal (convex side) surfaces. Why do both surfaces of retina require blood vessels? This query is valid because it is currently believed that cornea has no blood vessel supply because of an evolutionary mandate for it to be transparent, in order to enable light's entry uninterrupted onto the image forming retina. If that is the case, why are the rod/cone cells tucked away at the distal side of the retina? Did evolution facilitate the rod-based detection of diffused light (9) so as to minimize DROS production? [Quoting Kono et al., (9): "Taken as a whole, our study is consistent with the hypothesis that the steps in the visual cycle are optimized such that the rod can serve as a highly sensitive dim light receptor."' In which case, would a simpler translucent membrane (Figure 3, bottom panel) fold near the lens not afford diffused light of lower intensities, rather than having varying blood vessels, nerve fibers, various cell types, etc., pose barricades to light in the retina itself, before it reaches the photoreceptor rod and cone cells?

3. Oxygen seems to have no roles in the direct visualization process in the classical perception. Why should the visual cycle have a high oxygen requirement and why should its absence lead to black outs? Why does the scheme not involve photochemically derived electrons, as photochemical processes are known to rest on such processes? If high light intensities give high amounts of DROS, does it not imply that lower light intensities give lower DROS?

4. The neuronal trigger is supposed to come from a conformation change of opsins in the rod/cone cells. How does this work, what is the trigger and how is it relayed/conducted from the ROS disks all the way to the optic nerve? We find that the current perceptions with ionic transports and enzymic cascades are too slow and fail to meet the basic criteria for enabling dynamic vision, with good spatio-temporal connectivity/coherence.

5. Barring small anatomical features such as fovea and blind spot, retina is mostly a concave surface (the inner side of an approximate sphere) with the number of rod/cone cells packed at $<2 \times 10^{5} / \mathrm{mm}^{2}$. That is, the diameter of a receptor cell corresponds to 30-60 arcsec. Even if we assumed that "one photon from one point could trigger at least one receptor" as the minimal requirement for a given point's visualization (which, we know for sure, is not the case!), we cannot explain how some persons could have acuity (or resolution) of $\sim 5 \operatorname{arcsec}$ (which is smaller than the receptor's dimensions!), when calculated with various distances, lighting, etc. (10).

The prevailing understanding of the visual cycle does not seem to be strongly supported by the histological realities of retina, and temporal-constraints seem to be overwhelming. It is difficult to understand the exact control of transducin and phosphodiesterase mediated pathways and how they lead to an immediate altering of the trans-membrane potential (TMP). Further, there is not one photo-electro-chemical event in such a scheme that could serve as a direct trigger for the propagation of an action potential along a neuron. Isomerization, conformational changes and protein binding events are merely physical processes; and these do not qualify for an electrochemical switching mechanism for the development of de-/ hyper-polarization (i.e. significant change in temporal trans- 
membrane potential). Further, it does not make evolutionary/ functional sense that rhodopsins oligomerize (11).

\section{THE MURBURN MODEL FOR PHOTORECEPTION AT THE}

\section{EXISTENTIAL FUNCTIONING OF MAMMALIAN EYE}

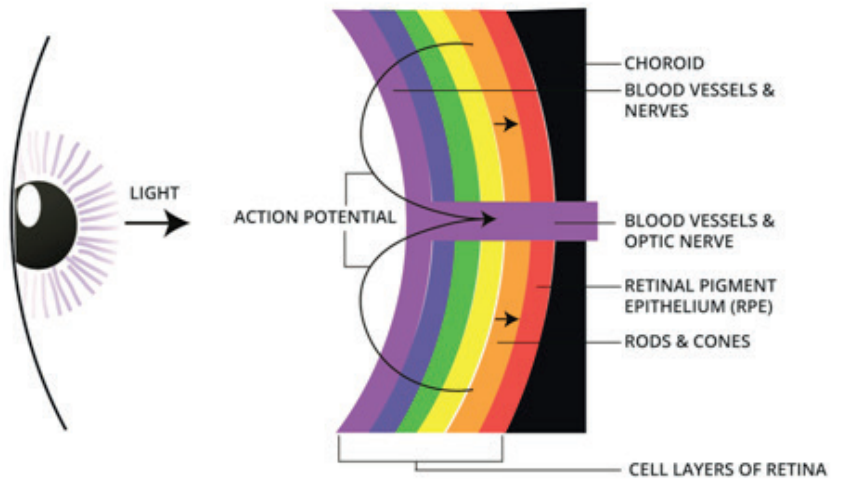

EVOLUTIONARY MANDATE OF CONVENTIONAL MODEL
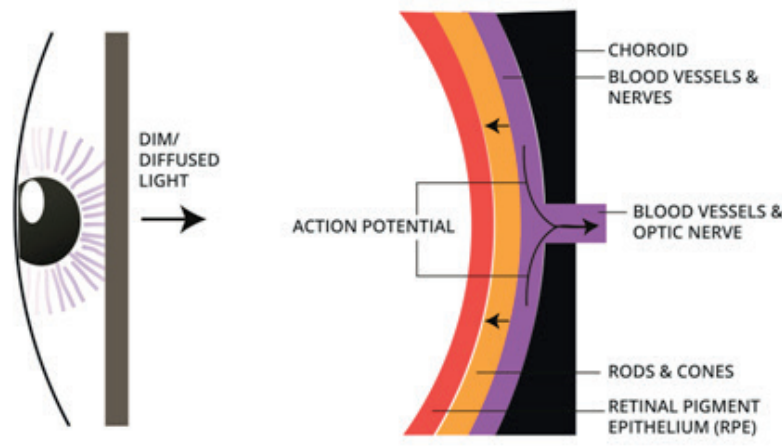

Figure 3. The existential and anticipated structures of retina, if the currently-held perceptions were operatively relevant. It does not make evolutionary/physiological sense that the photoreceptive rods/cones are located at the distal side of the retina. This leads to complexities in structure-function correlations. If rods and cones were the primary/sole photoreceptors, it would not serve to have the preceding intervening layers of neurons and the retinal architecture should have been similar to the one shown in the bottom panel, with reorientations of the rods/cones (the two small arrows in the orange layer) and RPE. Further, if eye's evolution was geared to detect and analyze diffused light for the sake of minimizing DROS production by rods, then it would have better served the eye to have a membrane present before or after the eye, to lower light entering the retina (beside the iris/pupil function; as show in bottom panel).

\section{RETINA, SPATIAL RESOLUTION AND SIGNAL RELAY TO BRAIN}

Figure 4 shows our proposed scheme at three different levels: (i) light's interaction with molecules leads to charge separation; (ii) the interfacial event leads to potential generation due to confinement of superoxide, leading to hyperpolarization and activation of opsin GPCR (transducin); and (iii) synapsenetworked neurons relay this minute charge across a finite distance to the brain (observed physically in the form of an action potential).

\section{EVIDENCE AND ARGUMENTS SUPPORTING THE NEW MURBURN MODEL}

1. Carotenoids (to which retinoids belong) are both photoand redox- active $(12,13)$. Therefore, activated rhodopsin can release an electron to be taken up by oxygen, and the DROS formed could also attack various types of retinoids within the milieu. Therefore, the theoretical premise for the reaction schemes shown in the top panel of Figure 4 is justified. The occurrence of DROS in retina have been documented time and again, although with the perception that they are deleterious to physiology $(14,15)$. Therefore, the prima facie evidence for DROS involvement in routine retina-physiology is established. A comparison of the architecture of three different systems (photosensitive rod/cone cells of retina, photosynthesizing chloroplasts and photorespiring cyanobacteria), as shown in Figure 5, demonstrates the commonality of stacked lipid membranes ensconcing nanopools of water. This structural organization minimizes free proton availability and utilizes the features of superoxide at the phospholipid membrane interface. Otherwise, protein and small molecules associations and interactions are more facile in free solution and much slower in lipids. With DROS mechanism, it makes more sense for proteins to oligomerize too, as seen from the murburn perspective.

2. We have traced the historical precedence in literature (16-19) and also demonstrated the theoretical, experimental and physiological viability of the proposal/fact that DROS (like superoxide) can sponsor non-specific phosphorylations in ambient redox metabolic environments $(3,20-24)$. Taking this account in the current context, the murburn model of signal transduction is ratified by the well-known phosphorylation of seven different ser/thr residues on rhodopsin $(25,26)$. This is also a very valid support for the activation of transducin by ROS by virtue of phosphorylation of the bound GDP. This is a much simpler and more probable explanation than GDP-GTP exchange (Figure 4). The surplus build of superoxide also 

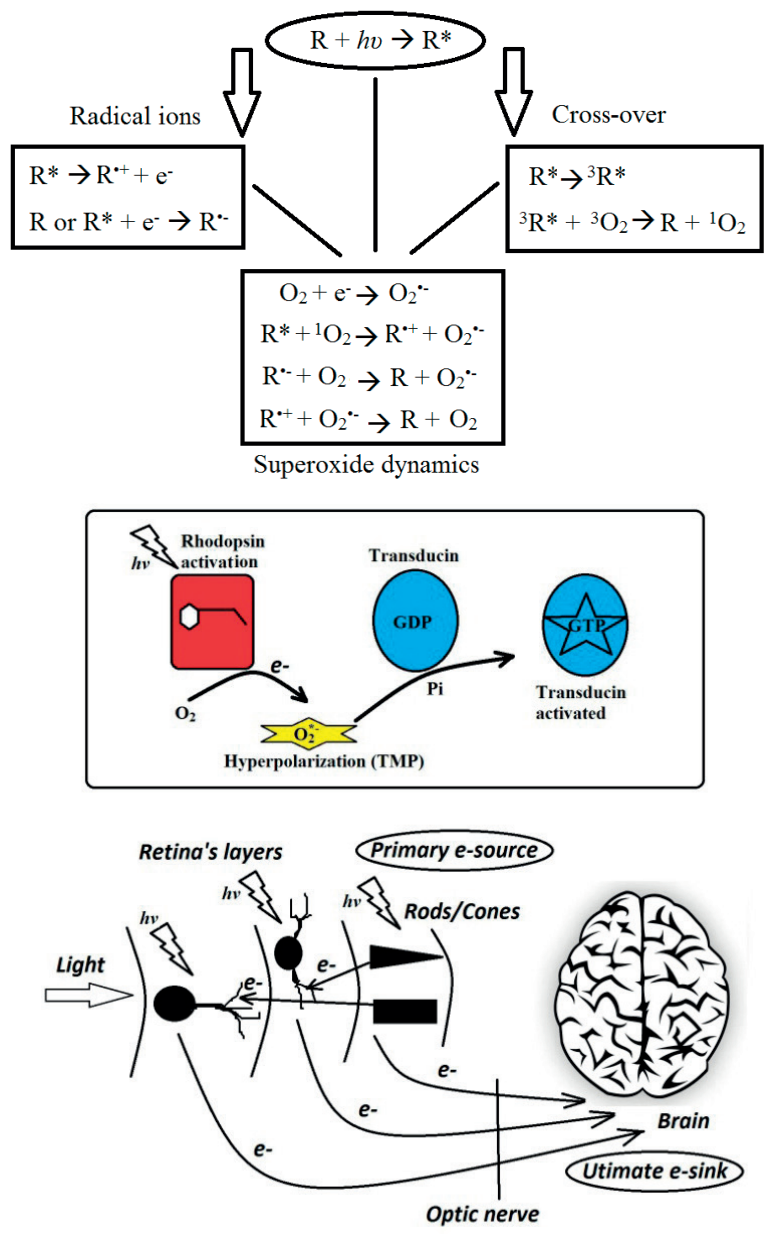

Figure 4. A simplified murburn model for physico-chemical transduction (charge separation leading to a potential generation), followed by signaling cascade and the overall connectivity for relay of signals. The top panel shows the photoreceptive charge separation leading to superoxide; the middle panel shows how superoxide formation can lead to hyperpolarization (negative charge surplus) and lead to phosphorylation of GDP bound to transducin (or at various loci of rhodopsin). The bottom panel shows that all layers of retina could be photosensitive, although the bottom layer is most efficient in charge separation owing to the stacking of membranes into disks (similar to the thylakoids of chloroplasts). Therefore, a minute and punctuated charge relay (with synapses serving as amplifiers along the path) across the neurons to the brain can result, leading to the transmission of a signal upon stimulation with light. Since the light perception occurs at multiple depths in retina, this can also afford better resolution and depth perception at the retina side itself (and depth perception may not be merely a trained routine of brain image reconstruction).

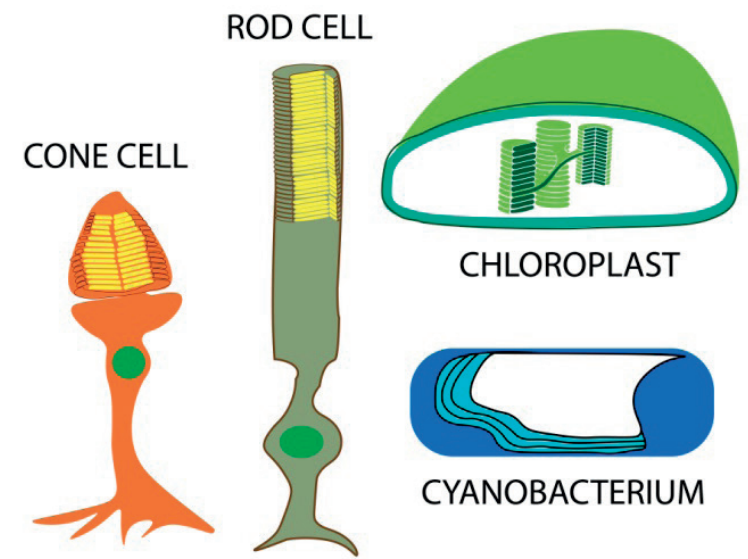

Figure 5. The stacking of nanopools of phospholipid stacked vesicles is a common strategy in rod/cone cells of retina and chloroplasts/cyanobacteria. The cells or organelle are not drawn to scale. Rod discs are micrometer dimensioned $\left(10^{3}\right.$ per rod), with $10^{5}$ rhodopsin per disk and there are $10^{8}$ rhodopsin per rod. Each disk is only $10 \mathrm{~nm}$ thick and 1 um wide (the rough absorption dimension of rod). They are cut open to show how nano-micro dimensioned lipid vesicles are stacked together for effective transduction of light for various purposes in different systems, with the intermediacy of superoxide (DROS).

explains the hyperpolarization phenomenon observed with light impingement.

3. Evolutionarily, melanopsin containing ganglion cells were the original photoreceptors in earlier life forms and it makes no sense why they should not be photosensitive in vertebrates. Our proposal that photoreception occurs at all levels in retina and they aid resolution and depth perception is supported by recent publications, which show that ganglion cells' melanopsin also contributes to vision (27).

4. As per the existing perception, sensing a photon necessitates cis-trans conversion of retinal and recycle of at least one rhodopsin molecule with the assistance of RPE (as shown in Figure 2). This has significant energy costs, as the cascades and recycling process are energy-intensive (keeping the pumps/ channels working, phosphorylation of components, etc.), with rods consuming about $10^{8} \mathrm{ATP} \mathrm{s}^{-1}$ in the dark (28). The light radiation incident on retina (in a room under normal reading/ visual conditions) is $\sim 2 \times 10^{8}$ photons per second (29). The number of rod/cone cells in the retina is $\sim 1 \times 10^{8}$ (30). Considering that the human eye can detect even a single photon (31), it must mean that a single photoreceptor (rod/cone cell) is capable of firing an action potential on its own, by the impinge- 
ment of a photon. Therefore, it is unimaginable that to process a single photon, the physiology must overwhelmingly waste its power reserves and keep chugging along with impossible rates for recycling. The involvement of DROS does not pose such energetic or kinetic issues.

5. If evolution had horned the structure/function of eye so that rod cells dealt with diffused light, then why do several carnivores have 'tapetum lucidum' (with components like riboflavins and zinc), which facilitates DROS dynamics? Surely, the earlier hypothesis is an energetic disadvantage because rods consume more ATP in dark, than in light (28).

\section{CONCLUSIONS}

We have presented here a brief critique on the prevailing understanding on the physiology of vision and proposed an alternative perspective in lieu. We do understand that invoking superoxide for constructive physiological outcomes would be aesthetically unappealing, given the literature in the context $(14,15,32)$. Our works have shown and argued that DROS are crucial to life. Besides, nitric oxide is used as a molecular messenger in cells! Further, the details of depth perception and roles of players like melatonin (33) are unaddressed yet in the new perspective. We shall shortly follow through with a more elaborate write-up on the ramifications of this topic. In the meanwhile, it would be really worthwhile receiving peers' criticisms and inputs on the viability or failure of this new idea, with respect to the known data.

\section{DECLARATIONS}

This work was powered by Satyamjayatu: The Science \& Ethics Foundation. We thank Ann Vinod for proofing the paper. The authors have no conflict of interests to declare.

\section{REFERENCES}

1. Sakmar TP, Huber T. Rhodopsin. Encyclopedia of Neuroscience 2009; 365-372. doi: 10.1016/B978-0080450469.00922-0

2. Kiser PD, Golczak M, Palczewski K. Chemistry of the retinal (visual) cycle. Chem Rev 2014; 114: 194-232. doi: $10.1021 / \mathrm{cr} 400107 \mathrm{q}$

3. Manoj KM, Manekkathodi A. Light's Interaction with Pigments in Chloroplasts: The Murburn Perspective, with Special Relevance to Carotenoids. OSF Preprints 2020. (In Press). doi: 10.31219/osf.io/wx4gv

4. Boycott BB, Dowling JE. Organization of the primate retina: Light microscopy, with an appendix: A second type of midget bipolar cell in the primate retina. Phil Soc Trans R Soc Lond B 1969; 255: 109-184. doi: 10.1098/ rstb.1969.0004

5. Berg JM, Tymoczko JL, Stryer L. Biochemistry. WH Freeman, 2002.

6. Lehninger A L, Nelson DL, Cox M. Principles of Biochemistry. Palgrave Macmillan Limited, 2004.

7. Voet D, Voet JG. Biochemistry. Wiley, 2011.

8. Masland RH, Martin PR. The unsolved mystery of vision. Curr Biol 2007; 17:R577-R582. doi: 10.1016/j. cub.2007.05.040.

9. Kono M, Goletz PW, Crouch RK. 11-cis- and all-transretinols can activate rod opsin: rational design of the visual cycle. Biochemistry 2008; 47:7567-7571. doi:10.1021/ bi800357b

10. Westheimer G. Visual hyperacuity. Prog Sensory Physiol 1981; 1:1-30.

11. Palczewski K. G protein-coupled receptor rhodopsin. Annu Rev Biochem 2006;75:743-67. doi: 10.1146/annurev.biochem.75.103004.142743.

12. Han R-M, Zhang JP, Skibsted LH. Reaction dynamics of flavonoids and carotenoids as antioxidants. Molecules 2012; 17:2140-2160.

13. Konovalova TA, Kispert LD, Konovalov VV. Photoinduced electron transfer between carotenoids and solvent molecules. J Phys Chem B 1997; 101:7858-7862.

14. Nita M, Grzybowski A. The role of the reactive oxygen species and oxidative stress in the pathomechanism of the age-related ocular diseases and other pathologies of the anterior and posterior eye segments in adults. Oxid Med Cell Longevity 2016; 3164734. doi: 10.1155/2016/3164734

15. Rohowetz LJ, Kraus JG, Koulen P. Reactive oxygen species-mediated damage of retinal neurons: Drug development targets for therapies of chronic neurodegeneration of the retina. Int J Mol Sci. 2018; 19:3362. doi: 10.3390/ ijms19113362.

16. Cohn M, Drysdale GR. A study with O18 of adenosine triphosphate formation in oxidative phosphorylation. $J$ Biol Chem 1955; 216:831-846.

17. Mironova GD. Metmyoglobin and peroxide compounds in mitochondria. In: Mitochondria. Structure and Functions in Norm and Pathology. pp 169-173. Nauka, Moscow. 1971

18. K. Mailer, Superoxide radical as electron donor for oxidative phosphorylation of ADP. Biochem. Biophys. Res. Comm. 1990; 170: 59-64. 
19. Tyszkiewicz E, Roux E. Role of the superoxide anion $\left(\mathrm{O}_{2}{ }^{-}\right)$ and hydroxyl radical $(\mathrm{OH} \cdot)$ in ATP synthesis obtained with spinach chloroplasts in darkness. In: Progress in Photosynthesis Research. Biggens J editor. Vol. III, 213-216. Martinus Nijhoff Publishers, 1987

20. Manoj KM, et al. Aerobic respiration: proof of concept for the murburn perspective. J Biomol Struct Dyn 2019; 37:4524-4556. doi:10.1080/07391102.2018.1552896

21. Manoj KM, et al. Chemiosmotic and murburn explanations for aerobic respiration: predictive capabilities, structure-function correlations and chemico-physical logic. Arch Biochem Biophys. 2019; 676:108128. doi: 10.1016/j.abb.2019.108128

22. Manoj KM, Bazhin N. Murburn precepts of aerobic respiration and homeostasis. OSF Preprints 2019. doi:10.31219/osf.io/hx4p9

23. Manoj KM, et al. Acute toxicity of cyanide in aerobic respiration: Theoretical and experimental support for murburn explanation. Biomol Concepts 2020a; 11:32-56. doi:10.1515/bmc-2020-0004

24. Manoj KM, et al. Murburn precepts for the light reaction of oxygenic photosynthesis. OSF Preprints 2020b; doi: 10.31219/osf.io/95brg

25. Wilden U, Kühn H. Light-dependent phosphorylation of rhodopsin: number of phosphorylation sites. Biochemistry 1982;21(12):3014-22. doi: 10.1021/bi00541a032
26. Maeda T, Imanishi Y, Palczewski K. Rhodopsin phosphorylation: 30 years later. Prog Retin Eye Res 2003; 22:41734. doi: 10.1016/s1350-9462(03)00017-x

27. Zele AJ, Feigl B, Adhikari P, et al. Melanopsin photoreception contributes to human visual detection, temporal and colour processing. Sci Rep 2018; 8: 3842. doi: 10.1038/ s41598-018-22197-w

28. Okawa H, Sampath AP, Laughlin SB, Fain GL. ATP consumption by mammalian rod photoreceptors in darkness and in light. Curr Biol. 2008; 18:1917-1921. doi:10.1016/j. cub.2008.10.029

29. Yu H-H. How many photons get into your eyes? 2016; https://medium.com/cortically-magnified/estimating-thenumber-of-photons-that-hit-the-eye-c0208e7e0b64

30. Purves D, Augustine GJ, Fitzpatrick D, et al. Neuroscience, $2^{\text {nd }}$ edition. Sunderland, MA, Sinauer Associates, 2001.

31. Tinsley JN, Molodtsov, M, Prevedel, R et al. Direct detection of a single photon by humans. Nat Commun 2016; 7:12172. doi: $10.1038 /$ ncomms 12172

32. Mittag TW, Bayer AU, La VM. Light-induced retinal damage in mice carrying a mutated SOD I gene. Exp Eye Res 1999; 69(6): 677-683. doi: 10.1006/exer.1999.0748

33. Tosini G, Baba K, Hwang CK, Iuvone PM. Melatonin: an underappreciated player in retinal physiology and pathophysiology. Exp Eye Res 2012;103:82-89. doi:10.1016/j. exer.2012.08.009 